\title{
Handover Management for D2D Communication in 5G Networks
}

\author{
Wei Kuang Lai ${ }^{1}$, Chin-Shiuh Shieh ${ }^{2, *}$ (D) Fu-Sheng Chou ${ }^{1}$, Chia-Yu Hsu ${ }^{1}$ and Meng-Han Shen ${ }^{1}$ \\ 1 Department of Computer Science and Engineering, National Sun Yat-sen University, \\ Kaohsiung 80424, Taiwan; wklai@cse.nsysu.edu.tw (W.K.L.); fusheng699@gmail.com (F.-S.C.); \\ alde0130@gmail.com (C.-Y.H.); eerica860tw@gmail.com (M.-H.S.) \\ 2 Department of Electronic Engineering, National Kaohsiung University of Science and Technology, \\ Kaohsiung 80778, Taiwan \\ * Correspondence: csshieh@nkust.edu.tw; Tel.: +886-937-316833
}

Received: 11 June 2020; Accepted: 23 June 2020; Published: 26 June 2020

\begin{abstract}
This study addresses the handover management issue for Device-to-Device communication in fifth-generation (5G) networks. The Third Generation Partnership Project (3GPP) drafted a standard for proximity services (ProSe), also named device-to-device (D2D) communication, which is a promising technology in offering higher throughput and lower latency services to end users. Handover is an essential issue in wireless mobile networks due to the mobility of user equipment (UE). Specifically, we need to transfer an ongoing connection from an old E-UTRAN Node B (eNB) to a new one, so that the UE can retain its connectivity. In the data plane, both parties of a D2D pair can communicate directly with each other without the involvement of the base station. However, in the control plane, devices must be connected to the eNB for tasks such as power control and resource allocation. In the current standard of handover scheme, the number of unnecessary handovers would be increased by the effect of shadowing fading on two devices. More important, the handover mechanism for D2D pairs is not standardized yet. LTE-A only considers the handover procedure of a single user. Therefore, when a D2D pair moves across cell boundaries, the control channels of the two UEs may connect to different base stations and result in increased latency due to the exchange of D2D related control messages. Hence, we propose a handover management scheme for D2D communication to let both parties of a D2D pair handover to the same destination eNB at the same time. By doing so, the number of unnecessary handovers, as well as the handover latency, can be reduced. In the proposed method, we predict the destination eNB of D2D users based on their movements and the received signal characteristics. Subsequently, we make a handover decision for each D2D pair by jointly factoring in the signal quality and connection stability. Expected improvement can be attained, as revealed in the simulation. Unnecessary handover can be avoided. Consequently, both UEs of a D2D pair reside in the same cell and, therefore, result in increased throughput and decreased delay.
\end{abstract}

Keywords: long term evolution advanced; Device-to-Device; handover; control signal; quality of service

\section{Introduction}

Due to the rapid evolution of technologies in recent years, the popularization of mobile devices such as smartphones, laptops, and tablets has resulted in immense changes in Internet connection scenarios. The wireless communication system enables ubiquitous connectivity for mobile users anytime and anywhere. More and more new mobile applications, such as high-definition audio, multimedia streaming, and augmented reality, have emerged, which has led to a tremendous demand 
for mobile data traffic. However, due to the scarce radio resources in wireless networks, an effective way to allocate radio resources while considering the throughput of the entire network and the quality of service (QoS) demand of each user equipment (UE) has become an important issue.

As a promising technique for fifth-generation (5G) cellular networks to solve the problem mentioned above, D2D communication has been first introduced in the Third Generation Partnership Project (3GPP) in Release 12, which adopts the concept of proximity services (ProSe) [1]. By allowing direct communication between two mobile devices without the involvement of an E-UTRAN Node $\mathrm{B}$ (eNB), device-to-device (D2D) communication can reduce loads of eNBs and the core network. Moreover, the transmission quality of UEs and the performance of the entire network would be improved as a result of a shorter transmission distance between two mobile devices.

However, expecting the mobility of users, seamless handover is necessary in order to provide ubiquitous communication when a user moves across a cell boundary. Most of the studies so far focus on the conventional Long Term Evolution-Advanced (LTE-A) handover procedure for a single cellular user [2-5]. The handover decision is mainly based on UEs' reports of received signal strength and quality, such as reference signal received power (RSRP), received signal strength indicator (RSSI), and reference signal received quality (RSRQ). In D2D communication, on the other hand, although the data can be directly transmitted among D2D devices, the connections between D2D UEs and eNBs are still needed in order to transmit the control signals so that the base stations can adjust the power of devices and allocate required resources to UEs.

Thus, there are two essential issues to be resolved in D2D handover management. First, it is possible to see that both devices of the same D2D pair are connected to different eNBs at the cell boundary. Such a configuration will generate additional latency due to the signal exchanges between the two eNBs using the $\mathrm{X} 2$ interface. Therefore, allowing a D2D pair to be associated with the same eNB bears a great advantage of scaling down signaling complexity and overhead among eNBs and D2D devices. Second, seeing UE movements and the possible shadow fading in a real environment, unneeded handovers would take place between two eNBs within a short period of time. The so-called ping-pong effect may happen when a D2D user moves around in the overlapped coverages of multiple eNBs or is obscured by trees, buildings, or other obstructions. In this aspect, the number of handovers incurred by the ping-pong effect also generates additional latency and degrades the system performance due to the reallocation of resources to the D2D users.

To jointly address these two issues, in this paper, we propose a novel D2D handover mechanism for every pair of D2D users to choose their handover target eNB based on their movements and make a handover decision based on the stability of the connections. In the proposed method, for the sake of reducing unnecessary handovers incurred by the shadow fading, a judicious selection of the handover target is indeed critical. In this aspect, we consider users' movements, including directional changes and distances to its neighbor eNBs, along with the signal-to-noise ratio (SNR) measured by each D2D UE. We first choose the most appropriate eNB as the potential handover target for each D2D pair from the candidate set of neighbor eNBs. This set will be updated at every time interval. Second, in order to let both users of the same D2D pair connect to the same eNB as much as possible and further reduce the number of handovers, we will make a handover decision for each D2D pair by jointly considering the RSRPs observed by both users to the original serving eNB and the target eNB.

The main contributions of this paper can be summarized as follows:

- Our approach ensures a more stable connection. In a realistic environment, due to the mobility of $\mathrm{UE}$ and the shadow fading, it is possible that the received signal strength is unstable. It results in frequent handovers, increased latency, and throughput degradation. In our proposed method, the historical moving directions and the RSRPs of both devices of the same D2D pair will be taken into account to pick up the handover target eNB.

- For finding the best target eNB for a D2D pair to jointly hand over two devices, the proposed method uses the RSRPs of both devices of the same D2D pair to define a utility function, which serves as a criterion used to choose the best handover target eNB. 
- Our scheme satisfies the transmission quality of each device and reduces the number of unnecessary handovers. This is because we make a handover decision for the same D2D pair by the connection quality and stability.

The rest of this paper is organized as follows. In Section 2, we provide a survey of related work in the literature. Then, our system model and the problem formulation are described in Section 3. The proposed approach is presented in Section 4. Subsequently, we analyze the performance of our proposed method, present the numerical results, and discuss the comparison with other approaches in Section 5. Finally, some conclusions will be drawn in Section 6.

\section{Related Works}

Many previous works in the literature have considered the problem of handover. According to the conventional LTE-A standard, a single cellular user can hand over from the serving eNB to the target eNB if the RSRP from the target eNB is higher than the RSRP from the serving eNB plus a hysteresis margin for a certain period, known as the time to trigger (TTT). This procedure is the so-called A3 event [6-8]. Therefore, some of the related works mainly focus on the configuration of the hysteresis margin and TTT. Priyadharshini et al. [9] employed different combinations of eNB coverage, hysteresis margin, and TTT in the urban and suburban areas to determine a better parameter setting. Likewise, Kollias et al. [10] observed the different failure rates in handover and connectivity for different TTTs to decide an optimal TTT value.

Numerous studies concentrate on the reduction of the number of handovers in order to decrease the latency. They first choose the target eNB based on the movements of devices and then decide whether or not to handover to the target based on the received signal condition. Wang et al. [11] proposed a handover mechanism to decide the best handover target for each user according to its historical movement records. In Chang et al. [12], a weighted-based handover scheme was proposed. The authors set the weights of candidate eNBs according to UE's bearing of movement. An eNB with the highest weight was picked up as the target. However, when a user makes a U-turn, the weights of some neighbor eNBs will still increase even though the user is moving away from such eNBs, which would result in additional unnecessary handovers. In Cheikh et al. [13], the Markov Chain is used to decide the connection probabilities to different eNBs based on the distances between a device and the neighbor eNBs. However, all the above works only consider the handover for a single cellular user.

Various works are devoted to D2D communication from different aspects. Sathya et al. [14] presents a solid study on the improvement of user SINR in LTE HetNets using D2D relay concept. There also exist studies focusing on the D2D handover management. Ouali et al. [15] provides a valuable insight from an analytic perspective into the D2D management problem. The D2D handover management problem with reference point group mobility (RPGM) model is modeled as a two-stage queuing network and a multi-dimensional Makov chain and used to evaluate the blocking and successful handover probability. Ouali et al. [16] reports a comprehensively comparative study on different D2D handover management techniques. They mathematically analyze the performance difference of selected solutions and conclude that a software defined network (SDN)-based solution outperforms others. In upholding the same belief that SDN-based solution is preferred, Yilmaz et al. [17] details the architecture and procedure deign, and demonstrates its superiority. Although an SDN-based approach is promising, a potential barrier is that SDN in not widely deployed. Li et al. [18] focuses on the handover of D2D clusters in fast-moving environment, such as highways. Their design takes advantage of D2D multicast in signaling and resource allocation. Simulation shows that the proposed approach can reduce communication interruption probability and handover latency. This works can be more complete if directional change can be considered.

As mentioned before, two devices of the same D2D pair are possibly connected to different eNBs at the cell boundary, which brings about additional latency. Therefore, some approaches have been proposed to cope with this problem. In Chen et al. [19], in order to let the users of the same D2D pair link to the same eNB as much as possible, the authors proposed a D2D handover mechanism. 
Specifically, for a D2D pair, if one of the devices has met the handover condition while the other has not, then it is allowed to postpone the handover decision until both devices have satisfied the handover condition. In case neither of the devices can satisfy the minimum requirement of a predefined RSRP threshold, the handover would be conducted immediately. However, this method entirely relies on the selected threshold, and the blocking problem may happen when a threshold is set too low. In Ouali et al. [20], the authors proposed two D2D handover schemes in which the movements of both devices of the same D2D pair were jointly considered. In the situation that both devices are moving in the same direction, the original serving eNB would hand them over together to the target eNB based on their measurements of received signal condition. Another scenario is that both devices are moving away from each other, and one of them has already handed over to a different eNB. In this case, the other UE would set up a countdown timer. This D2D pair would be disjointed into two independent cellular users if the timer countdowns to zero or the signal strength of the D2D link is lower than a predefined threshold.

\section{System Model and Problem Formulation}

\subsection{System Model}

Assuming there are $n$ macrocells in the network, we denote the set of eNBs as $N=\{1,2, \cdots, n\}$. Also, we assume that there are $d$ pairs of D2D users with $D U E_{i}^{1}$ and $D U E_{i}^{2}$ be the transmitter and the receiver of $i$-th D2D pair, respectively. For the received signal condition of each user, we use $R S R P_{k}^{i}$ to represent the RSRP received by $k$-th user from $i$-th eNB. In addition, we define $\varphi$ as the minimum requirement of RSRP for each device. Figure 1 shows the network environment under consideration in this paper. $D U E_{2}^{1}$ and $D U E_{2}^{2}$ have connected to different eNBs on their control channels. There are additional delays and overhead due to messages exchanges between these two eNBs. For simplicity and without loss of generality, we assume that each pair of D2D users is served by the same eNB in the beginning. Also, only the control plane is considered in this work; the data link between both users of the same D2D pair is out of the scope of this study [15].

In conventional trigger-based handover with the LTE A3 event, users inform the original serving eNB only when the handover conditions are met. Our method, however, let each user periodically report its measurement of received signals from its neighbor eNBs. The original serving eNB makes the handover decision for each D2D pair based on the channel conditions of both devices of the same D2D pair.

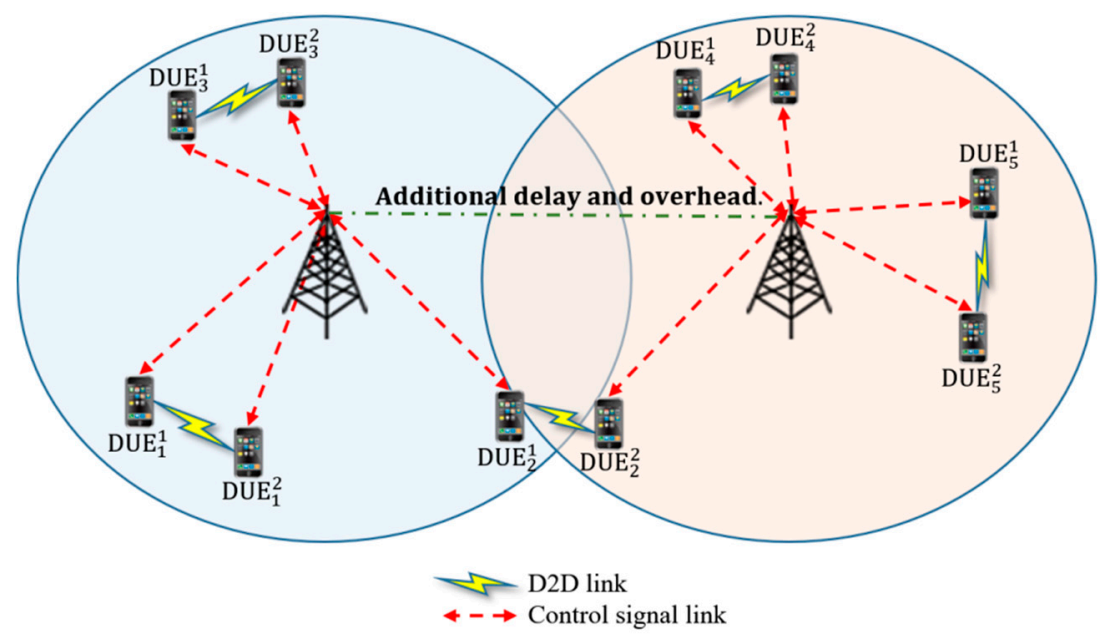

Figure 1. Network environment under consideration. 


\subsection{Problem Formulation}

For the sake of reduced latency, it is preferred to let both devices of each D2D pair connect to the same eNB as much as possible. This issue can be regarded as an optimization problem-maximizing the number of D2D pairs such that both parties connect to the same eNB. This optimization problem can be formulated as follows:

$$
\max \left(\sum_{i=1}^{d} X_{i}\right)
$$

subject to

$$
\begin{gathered}
\exists ! j \in\{1, \cdots, n\}, \alpha_{k}^{j}=1, \forall k \in\left\{D U E_{i}^{1}, D U E_{i}^{2}\right\}, \forall i \in\{1, \cdots, d\} \\
\alpha_{k}^{j} \cdot R S R P_{k}^{j} \geq \varphi, \forall j \in\{1, \cdots, n\}, \forall k \in\left\{D U E_{i}^{1}, D U E_{i}^{2}\right\}, \forall i \in\{1, \cdots, d\}
\end{gathered}
$$

where D2D UE is denoted as DUE. DUE $E_{i}^{1}$ and $D U E_{i}^{2}$ are the two UEs of the $i$-th D2D pair. $X_{m} \in\{0,1\}$ is an indicator indicating whether both users of the $i$-th D2D pair $\left\{D U E_{i}^{1}, D U E_{i}^{2}\right\}$ connected to the same eNB or not. $X_{i}=1$ means that both $D U E_{i}^{1}, D U E_{i}^{2}$ connect to the same eNB, and $X_{i}=0$ otherwise. Also, $\alpha_{k}^{j}$ is a Boolean variable where $\alpha_{k}^{j}=1$ indicates that there is a control link between user $k$ and eNB $j$. The $R S R P_{k}^{j}$ is the RSRP received at user $k$ from eNB $j$. In the optimization problem (1), the objective is to let both devices of each D2D pair connect to the same eNB as much as possible. Constraint (2) specifies that every user connects to exactly one eNB. Constraint (3) guarantees the signal quality for each user in order to maintain the reliability of the control signals.

It is obviously that the control cost (or signaling cost, or connection cost) when both users of a D2D pair connect to the same eNB, denoted by $\hat{C}$, is lower than the control cost when two parties of a D2D pair connect to different eNBs, denoted by $\check{C}$. It can be shown that a configuration with more D2D pair connected to the same eNB will result in a lower control cost, as shown in the following theorem.

Theorem 1. A configuration with a lower connection cost would have more D2D pairs connected to the same eNB.

Proof. Suppose there are two different configurations and one configuration has a connection cost of $l$ and the other has a connection cost of $\mathrm{m}$. Without loss of generality, let $l>m$. We prove by contradiction and assume that the configuration with the connection cost $l$ has more D2D pairs connected to the same eNB.

If there are in total $n$ D2D pairs, there are at most $n$ pairs of D2D devices to the same eNB, and there are at least zero D2D connection pair to the same eNB.

Let $S=\left\{\left(d_{11}^{S}, d_{12}^{S}\right),\left(d_{21}^{S}, d_{22}^{S}\right), \cdots,\left(d_{s 1}^{S}, d_{s 2}^{S}\right)\right\}$ denote the set of D2D pairs that each pair of D2D devices is connected to the same eNB, $T=\left\{\left(d_{11}^{T}, d_{12}^{T}\right),\left(d_{21}^{T}, d_{22}^{T}\right), \cdots,\left(d_{t 1}^{T}, d_{t 2}^{T}\right)\right\}$ denote the set of D2D pairs that each pair of D2D devices is not connected with the same eNB, and $N=S \cup T, n=s+t$. Let each pair of D2D devices require a connection cost of $\hat{C}$, when this pair belongs to set $S$ and would require a connection cost of $\breve{C}$ when this pair belongs to set T. Plainly, $\breve{C}>\hat{C}$ since whenever a pair of D2D devices is with the same eNB, there would not require communication overhead between two neighboring eNBs and all communication signals are from the same BS. Then, the total connection cost TC can be calculated as follows:

$$
T C=s \cdot \hat{C}+t \cdot \check{C}
$$

Since $n=s+t$ the above equation shows that the lower the total connection cost is, the more the D2D pairs are connected to the same BSs, which leads to a contradiction to the assumption. Therefore, the theorem is proved.

The optimization problem in (1) can be approached by exhaustive search as follows. For $d$ D2D pairs and $n$ eNBs, each user can connect to one and only one of $n$ eNB. There are in total $(n)^{2 d}$ possible 
configurations. We can examine the feasibility of configurations one by one and count the number of D2D pairs having both parties connected to the same eNB. The complexity of such an approach is $O\left(n^{d}\right)$. Since the order of the complexity is exponential, the optimization problem cannot be solved solely by a primal entity in a practical system due to its complexity. Even more challenging is the mobile and dynamic nature of the system as in operation. Therefore, we propose a novel D2D handover mechanism for every pair of D2D users to choose their potential handover target eNB. The proposed approach, with complexity $O(d \cdot n)$, aims to reduce unnecessary handover and let both parties of a D2D pair connect to the same eNB.

\section{Proposed Approach}

Our objective is to reduce the unnecessary handovers caused by the possible ping-pong effect while keeping both users of the same D2D pair connected to the same eNB. Our proposed method consists of two phases-(1) choosing the appropriate target eNB as the potential handover target for each pair of D2D users and (2) deciding whether or not to hand over to the target eNB.

\subsection{Choosing Handover Target}

According to the current 3GPP handover standard, each device can amend its transmission quality by connecting to the eNB with the best-received signal status. However, in the realistic urban environment, there are obstacles such as buildings and trees. When a user is moving around in the overlapped area of multiple eNBs, the ping-pong effect may still happen due to possible shadow fading. This effect introduces additional transmission latency and further decreases system performance. Therefore, we should carefully choose the handover target, considering not only each user's received signal condition but also its movement.

Moreover, since both devices of the same D2D pair may connect to different eNBs at the cell boundary, which results in additional transmission latency, it is beneficial to let both users of the same D2D pair connect to the same eNB.

Therefore, to meet these goals, we would jointly consider the movements and the received signal of both users of the same D2D pair in order to choose the most appropriate eNB for each D2D pair as its potential handover target. Thus, by forcing both users of the same D2D pair to consider the same eNB as their handover target, we alleviate the latency caused by unnecessary handovers and avoid additional overhead generated between different eNBs. Consequently, in this section, we will propose a criterion for choosing the potential handover target for each D2D pair.

First, there may be multiple eNBs that can be the prospective handover targets for a mobile device during its movement. At each time slot, we have to maintain a list of candidate target eNBs and select the best of them to be the potential handover target for each D2D pair. Thus, we define a directional counter $D_{\text {counter }}{ }^{m, i}(t)$ as a counting value for $i$-th D2D pair with respect to $m$-th eNBs at time slot $t$. Also, we use $\Gamma_{i}$ to denote a set that consists of candidate targets for $i$-th pair of D2D users. Users may report their locations back to their serving eNBs at each time slot by utilizing the global positioning system (GPS) or other network-assisted positioning services. We can find out whether a user is approaching a neighbor eNB by merely observing the distance changes between this user and the eNB. Specifically, at time slot $t$, when a user $k$ (where $k \in\left\{D U E_{i}^{1}, D U E_{i}^{2}\right\}$ ) is approaching eNB $m$, the corresponding $D_{\text {counter }}$ value will be added by one (i.e., $\left.D_{\text {counter }} m, i(t)=D_{\text {counter }} m(t-1)+1\right)$. Note that $D_{\text {counter }}{ }^{m, i}(0)=0, \forall i \in\{1,2, \cdots, d\}, \forall m \in\{1,2, \cdots, n\}$. Also, eNB $m$ will be added to $\Gamma_{i}$ if it has not been added yet. Therefore, for an eNB with maximum $D_{\text {counter }}$ value with reapect to a D2D pair, the connection quality between them is supposed to be the most stable among all connections between other eNBs and that D2D pair. Thus this eNB would consequently be chosen as the handover target.

Second, assuming that there may be multiple eNBs in the set of candidate targets for a D2D pair with the same largest $D_{\text {counter }}$ value, another criterion is given for choosing the best target among them. In this aspect, we define a utility function in which the received signal conditions of both devices of the same D2D pair are considered. Specifically, the best target eNB should allow the shortest 
transmission time for both users of the same D2D pair to transmit their control signals. As each device will periodically report its received signal condition in terms of RSRP from all neighbor eNBs back to its serving eNB, we can denote the reported set of RSRPs for a device as follows:

$$
R S R P_{k}^{\text {report }}=\left\{R S R P_{k}^{1}, R S R P_{k}^{2}, \cdots, R S R P_{k}^{n}\right\}
$$

where $k \in\left\{D U E_{i}^{1}, D U E_{i}^{2}\right\}$ and $i \in\{1,2, \cdots, d\}$. Note that for each device, if the received signal power from certain eNB is too low to be detected, the corresponding RSRP value would be set to zero. Thus, we can calculate the SNR for each user $k$ with respect to an eNB $m$ by

$$
S N R_{k}^{m}=\frac{R S R P_{k}^{m}}{P_{\text {noise }}}
$$

where $m \in\{1,2, \cdots, n\}$ and $P_{\text {noise }}$ is the additive white Gaussian noise (AWGN) power. With the SNR value for each user with respect to each eNB, we can first calculate the transmission capacity, $C_{k}^{m}$, according to Shannon [21] and then further obtain the transmission time on the control channel for each user $k$ with respect to the eNB $m$, i.e.,

$$
\begin{gathered}
C_{k}^{m}=B_{k}^{m} \times \log _{2}\left(1+S N R_{k}^{m}\right) \\
T_{x}{ }^{m, k}=\frac{S_{k}}{C_{k}^{m}}
\end{gathered}
$$

where $B_{k}^{m}$ is the bandwidth allocated from eNB $m$ to user $k$, and $S_{k}$ is the size of the control signals required by user $k$. However, due to the equal size of control signals received by both users of the same D2D pair and the same bandwidth allocated to them, we can define our utility function for $i$-th pair of D2D users with respect to eNB $m$ by simply using SNR values of $D U E_{i}^{1}$ and $D U E_{i}^{2}$ as follows:

$$
\text { Utility }_{i}^{m}=\frac{1}{\log _{2}\left(1+S N R_{D U E_{i}^{1}}^{m}\right)}+\frac{1}{\log _{2}\left(1+S N R_{D U E_{i}^{2}}^{m}\right)}
$$

Thus, if there are multiple eNBs with the same largest $D_{\text {counter }}$ value to a D2D pair, we then allow the eNB with the largest utility value to be the potential handover target of such a D2D pair. In addition to multiple eNBs with the same largest $D_{\text {counter }}$ value, there might be a possible outage for a device within a D2D pair, which is caused by shadow fading or being too far away from the original serving eNB during its moving process. For this case, we would also use the utility function to determine the new serving eNB for the D2D pair.

Moreover, due to the random nature of user mobility, some devices could change their directions or even make a U-turn during their movements. This would lead to them to be more distant from their handover candidates and further generate some mismatches in their list of candidate targets. To this issue, we update the list of candidate targets for each D2D pair by tracking each user's moving process. Specifically, we first define a threshold in terms of turning angle $\rho$, and two moving vectors $\overrightarrow{V_{k}^{\text {old }}}$ and $\overrightarrow{V_{k}^{n e w}}$ as the initial moving direction and the current moving direction for user $k$, respectively. Then, for a user $k$ at time slot $t$, we can use the dot product of $\overrightarrow{V_{k}^{\text {old }}}$ and $\overrightarrow{V_{k}^{\text {new }}}$ to calculate its current turning angle $\theta_{k}(t)$ as follows:

$$
\theta_{k}(t)=\cos ^{-1}\left(\frac{\overrightarrow{V_{k}^{\text {old }}} \cdot \overrightarrow{V_{k}^{\text {new }}}}{\| \overrightarrow{V_{k}^{\text {old }}\|\cdot\| \overrightarrow{V_{k}^{\text {new }}} \|}}\right)
$$


Figure 2 illustrates an example of a user's moving process, where $P_{k}(t)$ is the position of user $k$ at time slot $t$. Thus, for a user $k$ at time slot $t$ where $k \in\left\{D U E_{i}^{1}, D U E_{i}^{2}\right\}$. If $\theta<\rho$, it implies that the turning angle of user $k$ is not large enough to cause the mismatches in $\Gamma_{i}$. Thus we only need to update the corresponding $D_{\text {counter }}$ values and add the appropriate eNBs to $\Gamma_{i}$ as mentioned before. Conversely, if $\theta \geq \rho$, we then set $\Gamma_{i}$ to $\varnothing$ in order to avoid any possible mismatches in the handover candidate list.

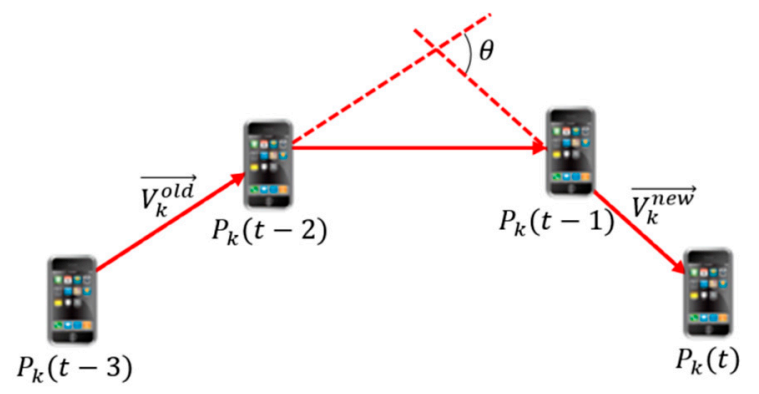

Figure 2. An example of a user's moving process.

According to the above rule, we design Algorithm 1 for the selection of a handover target for the $i$-th pair of D2D users. We use $C_{i}(t)$ to indicate the set of eNBs to which the $i$-th D2D pair is approaching and use $d_{k}^{m}(t)$ to represent the distance between user $k$ and eNB $m$ at time slot $t$.

Here, we briefly discuss our proposed algorithm. In Algorithm 1, for $i$-th pair of D2D users, Lines 4-6 handle the possible outage caused by shadow fading or being too far away from the original serving eNB. We use our utility function to guide both users to re-connect to a new serving eNB.

From Line 7 to Line 15, we have the historical position records of both users. The turning angle with respect to the initial moving direction for each user can be calculated accordingly. Subsequently, from Line 16 to Line 21, the turning angles of both users can be obtained. We then examine whether any user's turning angle is larger than the predefined threshold. If such a condition happens, we reset the set of handover candidates to empty and start a new assessment of the handover candidates.

In addition, we would observe the proximity of each user with respect to each eNB to update the corresponding $D_{\text {counter }}$ values and have appropriate new eNBs added to the list, as seen from line 23 to line 33. Consequently, from Line 36 to Line 38 , we first choose the eNB with the largest $D_{\text {counter }}$ value to be the handover target. While there may be multiple eNBs satisfying this condition, we further choose the eNB with the largest utility to be the handover target. Moreover, after choosing the handover target, it is necessary to make the decision about whether or not to hand over to the target. This handover decision process, referring to Line 35 to Line 39, will be discussed in the next section. Also, after the D2D pair successfully hand over to the target, some related parameters should be refreshed, which can be seen from Line 40 to Line 45 .

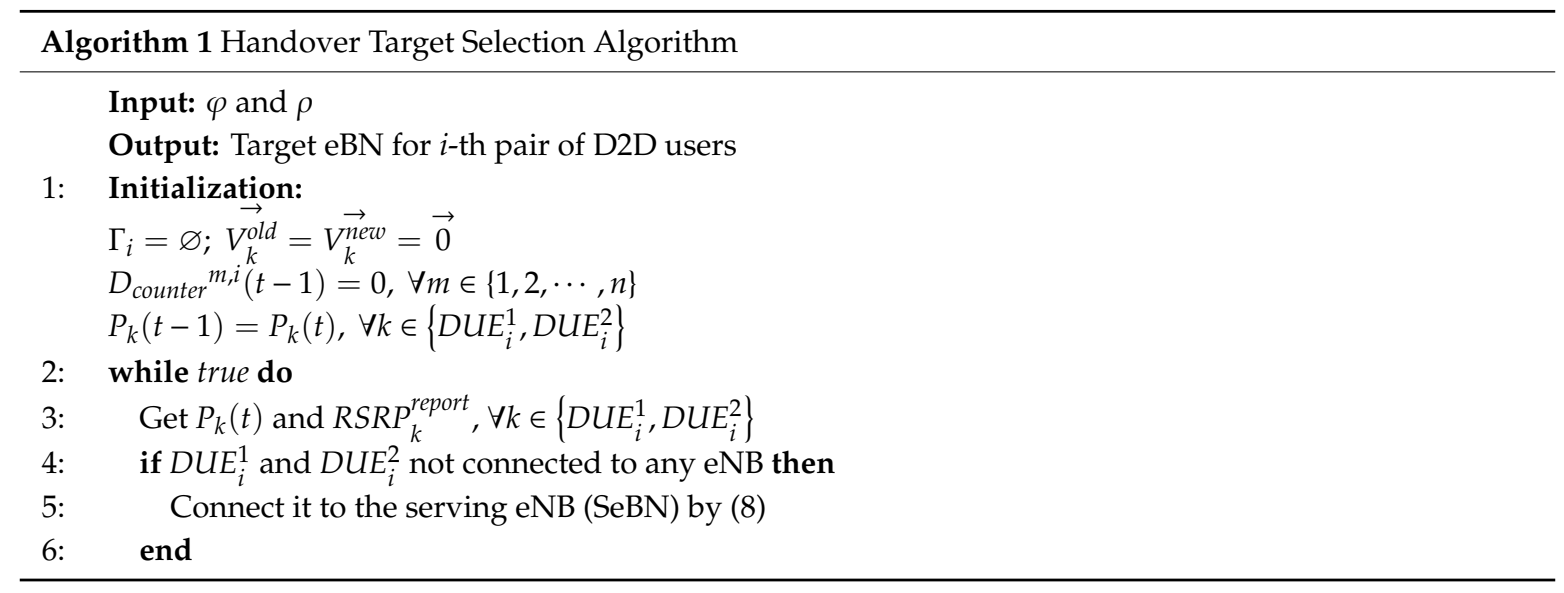




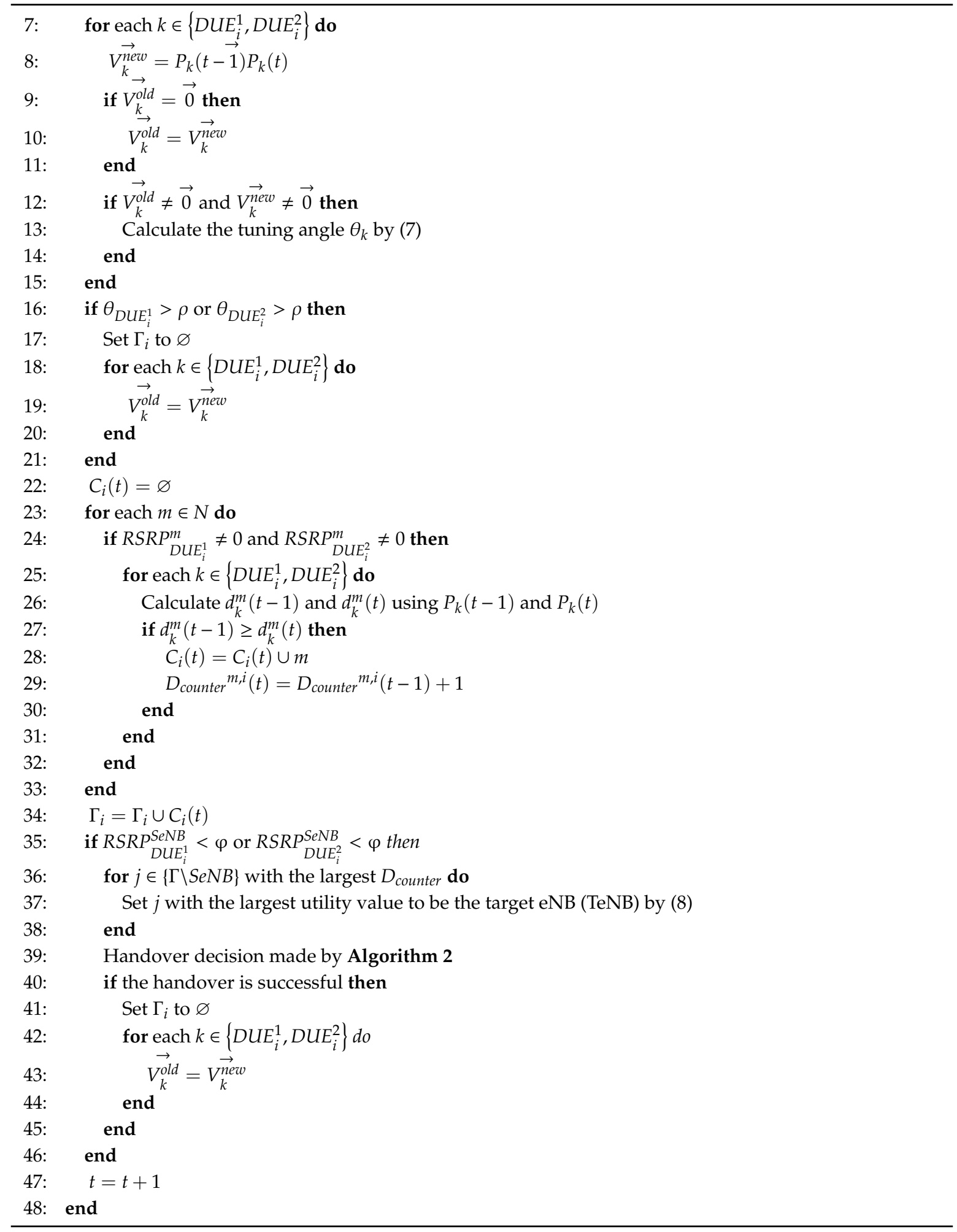

\subsection{Handover Decision}

After we select the potential handover target eNB for each D2D pair by jointly considering each user's movement and the received signal conditions of both users of the same D2D pair, we then need to decide whether or not to handover the D2D pair to the chosen target.

As mentioned before, the objective of this work is to reduce the unnecessary handovers caused by possible ping-pong effect and to ascertain both users of the same D2D pair to be with the same eNB. 
Since the latter and part of the former are achieved by choosing a single handover target eNB for each pair of D2D users and by jointly taking into account their movements and received signal conditions. In this section, we will make the handover decision for each D2D pair by factoring in the RSRPs of both users of a D2D pair so as to further reduce the unnecessary handovers and to ensure the stability of a connection.

As we have each user report its RSRPs received from all eNBs, we can thus examine the RSRPs received from the current serving eNB and the likely target eNB for the D2D pair. Specifically, for $i$-th pair of D2D users, since we have got $R S R P_{D U E_{i}^{1}}^{S e N B}, R S R P_{D U E_{i}^{2}}^{S e N B}, R S R P_{D U E_{i}^{1}}^{T e N B}, R S R P_{D U E_{i}^{2}}^{T e N B}$, and a predefined threshold of the minimum requirement of connection $\varphi$. Thus with the joint consideration of decreasing inappropriate handovers and raising the stability of the connection, we can make the decision on whether or not to hand over the D2D pair to the target eNB.

In this aspect, for $i$-th pair of D2D users, we first categorize the level of satisfaction regarding the serving eNB into the following three classes: (1) both users are satisfied with the minimum requirement of connection (i.e., $R S R P_{D U E_{i}^{1}}^{S e N B} \geq \varphi$ and $\operatorname{RSRP}_{D U E_{i}^{2}}^{S e N B} \geq \varphi$ ), (2) either one of them has attained the minimum requirement of connection, but the other has not (i.e., $R S R P_{D U E_{i}^{1}}^{S e N B} \geq \varphi$ and $R_{D S R} P_{D U E_{i}^{2}}^{S e N B}<\varphi$, or $R S R P_{D U E_{i}^{1}}^{S e N B}<\varphi$ and $\left.R S R P_{D U E_{i}^{2}}^{S e N B} \geq \varphi\right)$, and (3) neither one of them has reached the minimum requirement of a connection (i.e., $\operatorname{RSR}_{D}^{S U N B}<\varphi$ and $\operatorname{RSRP}_{\mathrm{DUE}}^{S e N B}<\varphi$ ).

For the first class, both users of the same D2D pair can sustain their stability of connection by connecting to the original serving eNB. For the sake of bringing down redundant handovers, it is not suggested to hand over to the target eNB.

For the second class, if connecting both users of $i$-th D2D pair to the target eNB, we can further sort it into the following three subclasses: (1) both users can satisfy their minimum requirement of connection (i.e., $R S R P_{D U E_{i}^{1}}^{T e N B} \geq \varphi$ and $R S R P_{D U E_{i}^{2}}^{T e N B} \geq \varphi$ ), (2) either one of them can satisfy the minimum requirement of connection but the other cannot (i.e., $\operatorname{RSRP}_{D U E_{i}^{T}}^{T e N B} \geq \varphi$ and $\operatorname{RSRP}_{D U E_{i}^{2}}^{T e N B}<\varphi$, or $\operatorname{RSRP}_{D U E_{i}^{1}}^{T e N B}<\varphi$ and $R S R P_{D U E_{i}^{2}}^{T e N B} \geq \varphi$ ), and (3) neither one of them can satisfy the minimum requirement of connection (i.e., $R S R P_{D U E_{i}^{1}}^{T e N B}<\varphi$ and $R S R P_{D U E_{i}^{2}}^{T e N B}<\varphi$ ). Specifically, for the first subclass, since the target eNB is better than the original serving eNB in terms of satisfaction, we then hand over both users to the target eNB to advance their stability. For the second subclass, the satisfaction is the same for the D2D pair with regard to its target eNB and its original serving eNB. In order to determine which eNB is better in terms of stability of connection for both users of the D2D pair, we make the comparison according to their $D_{\text {counter }}$ values. Since whatever the D2D pair connects to, the unsatisfied user's received signal condition would probably not be too disparate from the satisfied one because of the short distance between the two users. Thus, with the joint consideration of stability of connection and avoidance of ping-pong effect, we would designate the eNB with the largest $D_{\text {counter }}$ value and its strength holding for a period of TTT to be the new serving eNB. For the third subclass, as the target eNB is worse than the original serving eNB, we will retain the connection to the original serving eNB to minimize unnecessary handovers.

For the third class, both users of the $i$-th D2D pair are connected to the target eNB. We can further classify this class into three subclasses: (1) both users can satisfy their minimum requirement of connection, (2) either one of them can satisfy the minimum requirement of connection but the other cannot, and (3) neither one of them can satisfy the minimum requirement of connection. For the first subclass and the second subclass, we would hand over the D2D pair to the target eNB due to the target eNB's advantage over the original serving eNB in satisfying both users. For the third subclass, the minimum requirement of connections of both users cannot be satisfied, no matter connecting to the target eNB or the original serving eNB. We can only choose the best eNB with which both users have better-received signal strength, as specified in the utility function (8). Thus if such eNB can keep its advantage for a period of TTT, it will be the new serving eNB for the D2D pair. 
The handover decision rules above are comprehensively shown in Algorithm 2, where the first class, second class, and third class are listed from Line 1 to Line 3, Line 5 to Line 20, and Line 21 to Line 37, respectively. Thus, with Algorithm 1 and Algorithm 2, for each D2D pair, we can obtain the best handover target by jointly considering the movements and the received signal conditions of both users.

Finally, as a result of Algorithm 1 and Algorithm 2, we can achieve our goal of reducing unrequired handovers caused by a possible ping-pong effect while assigning both users of the same D2D pair to the same eNB.

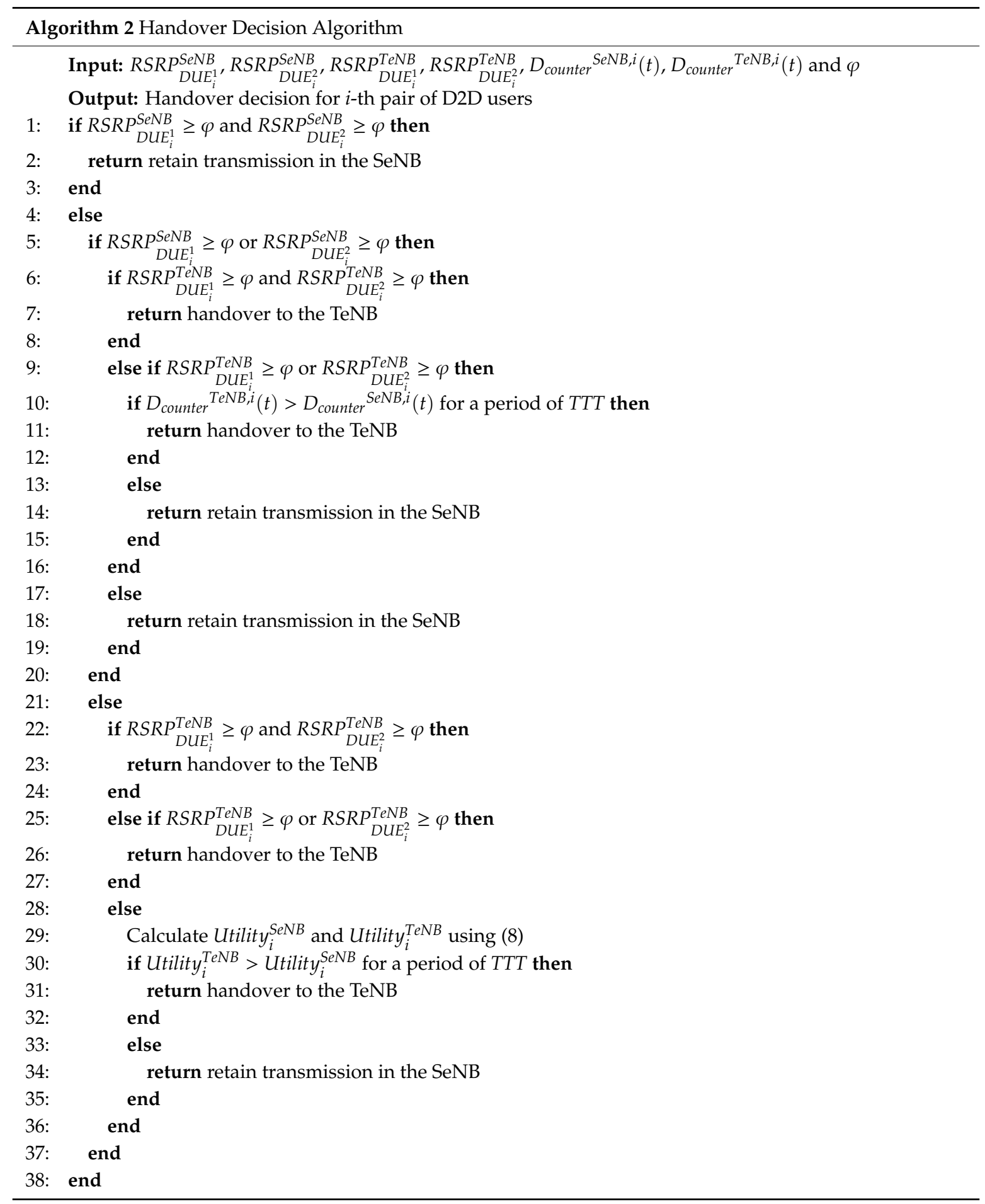




\subsection{Handover Procedure}

As we determine the target eNB and make the decision to hand over to it, the handover procedure will proceed accordingly.

The 3GPP standard of LTE-A specifies the handover procedure for a single cellular user [11]. The main difference with D2D handover is that, in the handover procedure of a D2D pair, the original serving eNB will issue the joint handover command to both users of the D2D pair. Both users detach from the previous serving eNB and synchronize with the target eNB after both the handover decision of the original serving eNB and the admission control of target eNB are made. After the synchronization for both users of the D2D pair with the target eNB, the target eNB would allocate radio resources for both users to sustain their D2D communication.

\subsection{Analysis of Computational Complexity}

In order to achieve a seamless handover for each pair of D2D users in a realistic environment, we need to ensure that our proposed methods are effective and efficient. Fortunately, for $n$ eNBs and $d$ D2D pairs, the time complexity of Algorithm 1 and Algorithm 2 for each pair of D2D users are only $O(n)$ and $O(1)$, respectively. Thus, the time complexity for each eNB to conduct the handover mechanism for every pair of D2D users in its coverage is at most $O(d \cdot n)$. Therefore, our proposed algorithms meet the real-time computational requirement in the real environment.

\section{Simulations}

\subsection{Parameter Settings and Network Topology}

To evaluate the system performance of the proposed algorithms, we consider a network environment consists of 19 eNBs, as shown in Figure 3. Also, there are 1-6 pairs of D2D users uniformly distributed in the coverage of each eNB. Table 1 shows the parameter settings used in the simulations.

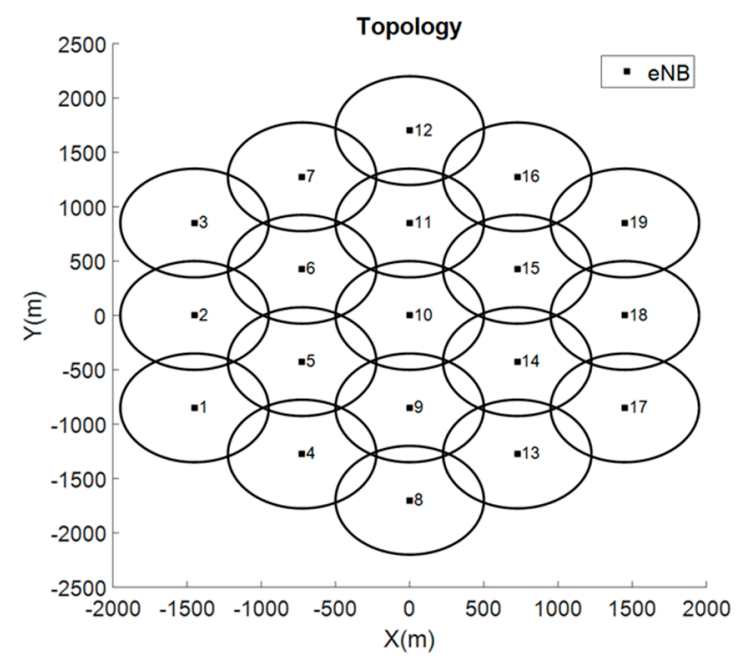

Figure 3. Network topology considered in the simulation.

Additionally, for the two DUEs of the same D2D pair, two mobility models are taken into account in the simulations. Particularly, the first one assumes that the fixed distance $(30 \mathrm{~m})$ is preserved between two DUEs of the same D2D pair during their movement. Also, they possess the same moving direction and velocity, while the moving direction would be changed every $3 \mathrm{~s}$. For this scenario, we name it as the fixed distance (FD) model. For the second scenario, we refer to the reference point group mobility (RPGM) model in Hong et al. [23]. It assumes that the movement of a group of users is mainly decided by the moving behavior of a central point in that group. Note that the moving direction of each user 
would be changed every $3 \mathrm{~s}$. Mathematically, the moving direction for a group member $l$ at time slot $\mathrm{t}$ can be expressed by

$$
\overrightarrow{V_{l}(t)}=\underset{V_{\text {group }}}{\vec{R}}(t)+R \overrightarrow{M_{l}}(t)
$$

where $\underset{V_{\text {group }}}{\rightarrow}(t)$ and $R \overrightarrow{M_{l}}(t)$ indicate the moving vector of the central point and a random biased vector with respect to the central point, respectively. In our simulation, when RPGM is used as the mobility model, each D2D pair $i$ would be viewed as a group. DUE $E_{i}^{1}$ would be regarded as the central point of this group, and $D U E_{i}^{2}$ can move around in the circular area around the central point with a radius of $30 \mathrm{~m}$.

Table 1. Simulation parameters.

\begin{tabular}{cc}
\hline Parameter & Value \\
\hline Bandwidth & $10 \mathrm{MHz}$ \\
Number of RBs & 50 \\
Number of eNBs & 19 \\
Number of D2D pairs & $1-6 \mathrm{per} \mathrm{eNB}$ \\
Radius of eNB & $500 \mathrm{~m}$ \\
Transmit power of eNB & $46 \mathrm{dBm}$ \\
Transmit power of UE & $23 \mathrm{dBm}$ \\
Path loss model [22] & eNB to UE: $128.1+36.7 \log d, d$ in km; UE to UE: $148+40 \log d, d$ in km \\
Shadow fading deviation & $8 \mathrm{~dB}$ \\
Distribution of D2D pair & Uniform \\
UE mobility model & Random direction \\
Speed of UE & $30,60,90,120 \mathrm{~km} / \mathrm{h}$ \\
Distance between DUEs & $5-30 \mathrm{~m}$ \\
$\varphi$ & $-80 \mathrm{dBm}$ \\
$\rho$ & 60 \\
A3 event margin & $3 \mathrm{~dB}$ \\
Time-to-Trigger (TTT) & $160 \mathrm{~ms}$ \\
Simulation rounds & 30 \\
Simulation time & $40 \mathrm{~s}$ \\
\hline
\end{tabular}

RBs: Resource Blocks; $\varphi$ : Threshold of minimal RSRP; $\rho$ : Threshold of turning angle.

Moreover, in this section, we also make comparisons among three methods. The first one is the conventional LTE-A handover standard in which the handover decision of a single cellular user is made according to the A3 event, which is referred to as the "standard" in our discussion. The second one is the method proposed in Ouali et al. [20], with which two DUEs of the same D2D pair find out the target eNB first, then both users jointly hand over to the target if both of them meet A3 event. However, if only one of them meets the A3 event, the satisfied one hands over to the target first, while the other stays with the original eNB until it meets the A3 event. The second method is referred to as "D-A3" in our discussion. The third scheme under consideration is our method. Our approach considers users' movements together with the received signal conditions for both DUEs of the same D2D pair. In order to evaluate the effect of individual mechanism, we conduct experiments considering only utility function as well as experiments considering both utility function and $D_{\text {counter }}$. The former is referred to as "D2D pairing (DP)," and the latter is referred to as " direction-counter-triggered D2D pairing (DTDP)" in our discussion. The signaling cost can be substantially reduced if both parties of a D2D pair connected the same eNB, DTDP and DP are therefore designed to let both parties of a D2D pair to handover together. For the sake of a fair comparison, the above-mentioned D-A3 will act the same in our simulations.

We will use the total number of handover, utility, satisfied ratio and average interruption time as our performance metrics for the comparative studies under different scenarios. In the following sections, we will analyze the numerical results in great detail. Particularly, the numerical results can be further divided into two parts. For the first part, the analysis is focused on the macro view of the 
simulated environment-that is, the average behavior of D2D pairs. Also, both of the aforementioned mobility models-FD and RPGM — would be examined. Subsequently, for the second part, in contrast to the first part, by observing the moving process of a single instance of D2D pair, we can thus analyze its performance from a micro point of view.

\subsection{Macro View of Numerical Results}

For the performance evaluation of the macro view of the system, we consider the following performance indicators: total number of handovers, the utility value measured using (8), and the satisfaction ratio which is assessed according to the predefined RSRP threshold $\varphi$. We observe the change in system performance by varying essential parameter settings, such as the number of D2D pairs and moving speed. Both mobility models—-the FD model and the RPGM model—are included in our simulations. Four approaches, namely Standard, D-A3, DP, and DTDP, are compared in our study to examine their relative merits and limitations.

Figure 4a shows the total number of handovers with respect to a different number of D2D pairs in the system. We can see that the proposed method has the fewest number of handovers compared to the other methods for both mobility models. Since the conventional standard only considers the received signal condition of a single cellular user, there is a high probability that the two DUEs of the same D2D pair will not simultaneously hand over to the same target when they are moving around in the area overlapped with multiple eNBs. This results in an additional number of handovers in the system. For the D-A3 method, both DUEs of the same pair will choose the same target eNB first and hand over to the target together when both of them have satisfied A3 events. DP only considers the received signal conditions of both DUEs, which does not take into account their movements. This will result in an additional number of handovers. On the other hand for the proposed DTDP, attributing to the $D_{\text {counter }}$ and the utility function, which accounts for both DUEs' movements and their received signal conditions, the better handover decision of a D2D pair to their preferred eNB can be realized. Now we examine the effects of different mobility models. Unlike FD which maintains a fixed distance (30 m) between both DUEs for each pair, the range between two devices of a D2D pair in RPGM is varied between 0 to $30 \mathrm{~m}$. Thus FD may have less probability to synchronously hand over both DUEs of the same pair. This lack results in about $16 \%$ to $21 \%$ more handovers than RPGM.

The impacts of the number of D2D pairs on the average utility value per D2D pair and the average satisfied ratio are shown in Figure $4 \mathrm{~b}$,c. The satisfied-D2D pair-ratio, simply referred to as the satisfied ratio hereafter, is defined as the ratio of D2D pairs that the RSRPs of both DUEs of that pair can attain the predefined threshold $\varphi$. The conventional standard may connect DUEs of a D2D pair to different eNBs. The average utility value per D2D pair and the satisfied ratio are calculated based on the received signal conditions for both DUEs when they are connecting to the same eNB. Therefore, the conventional standard is not included in the comparisons. As we can see from Figure $4 b, c$, the number of D2D pairs in the system and the type of mobility models do not significantly affect the result of average utility and satisfied ratio. This can be a result of the independence of D2D pairs and the sufficiency of resources in the network. We can still observe that the average utility value per D2D pair does really affect the result of satisfied ratio in the system. There is a trend that the higher the value of average utility per D2D pair, the better the satisfied ratio. The reason behind this phenomenon is that the eNB with a better received signal condition will be chosen using the proposed utility function (8). Moreover, the D-A3 method only chooses the target eNB that has the best RSRP to either one of the DUEs of the same pair. The other DUE may have a worse RSRP value with respect to the target. Thus, the average utility of D-A3 is inferior to our method. As for the performance difference between DTDP and DP, DTDP firstly considers $D_{\text {counter }}$ values rather than the utility values in the selection of the target eNB. This renders the average utility of DTDP slightly worse than that of DP, which only counts utility values in the handover mechanism. 



(a)
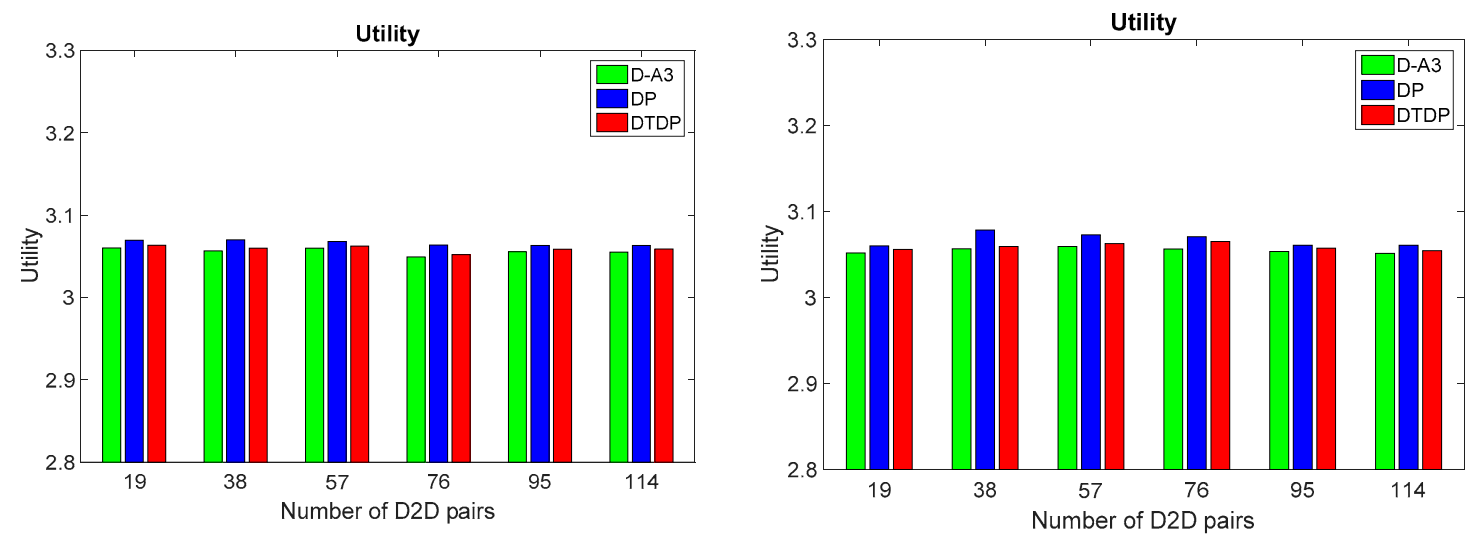

(b)
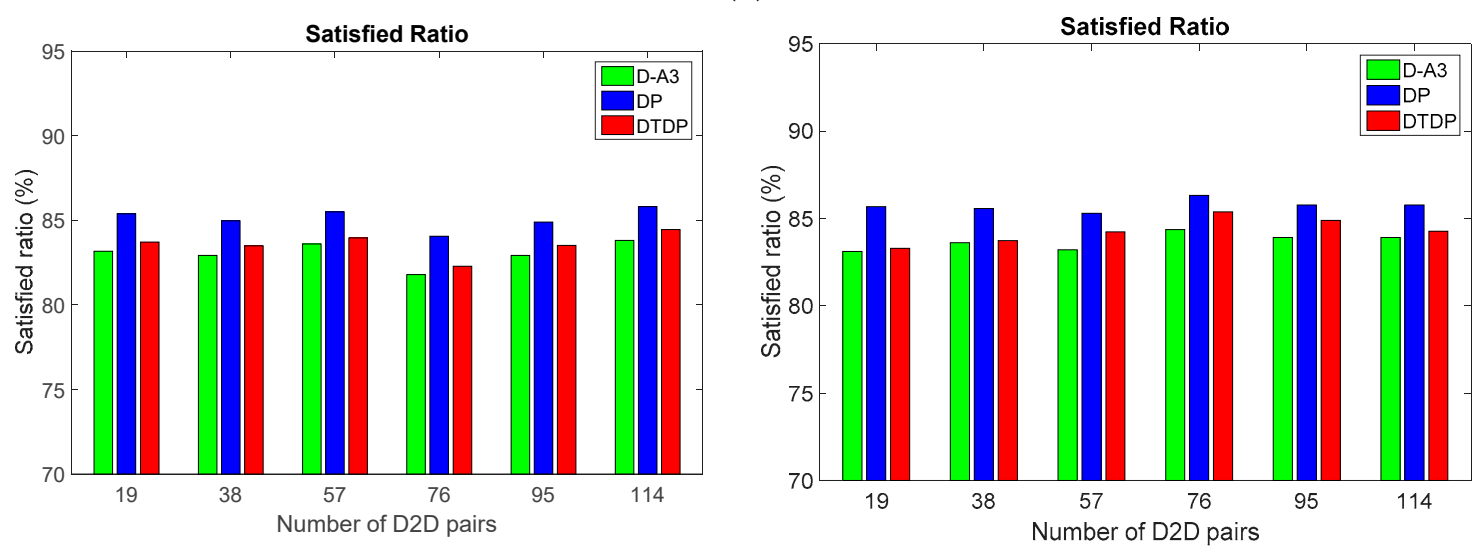

(c)

Figure 4. Total number of handover, utility, and satisfied ratio versus the number of device-to-device (D2D) pairs under different mobility models. The charts on the left and the charts on the right correspond to the fixed distance (FD) model and reference point group mobility (RPGM) model, respectively.

(a) Total number of handovers; (b) utility; (c) satisfied ratio.

Figure 5 a shows the impact of the moving speed of D2D users on the total number of handovers under both mobility models, FD and RPGM. As the moving speed of D2D users increases, there is a higher chance for D2D pairs to pass the areas overlapped with multiple eNBs. This results in a higher number of handovers. The conventional standard has the most number of handovers. It only considers the RSRP of each user, which may lead to different handover targets for the two DUEs of the same pair. For the DA-3 method, although the same target eNB is chosen by a pair of D2D users, the selected eNB might not be the best in minimizing the number of handovers. Thus additional handover times may still be generated. The proposed DTDP takes into account users' movement angels for the handover 
target choice while DP does not. With DTDP, there is a higher probability for each pair of D2D users to correctly determine whether to hand over to the new eNB or stay at current eNB. Therefore, the total number of handovers of DP is about $18 \%$ to $20 \%$ more than that of DTDP. The distance between two DUEs of the same D2D pair for RPGM is shorter than in FD. The total number of handovers of FD may be more than that of RPGM due to a considerably more difference in the RSRP values between two DUEs of the same pair. This causes more unstable received signal conditions for D2D pairs with respect to their connecting eNBs.
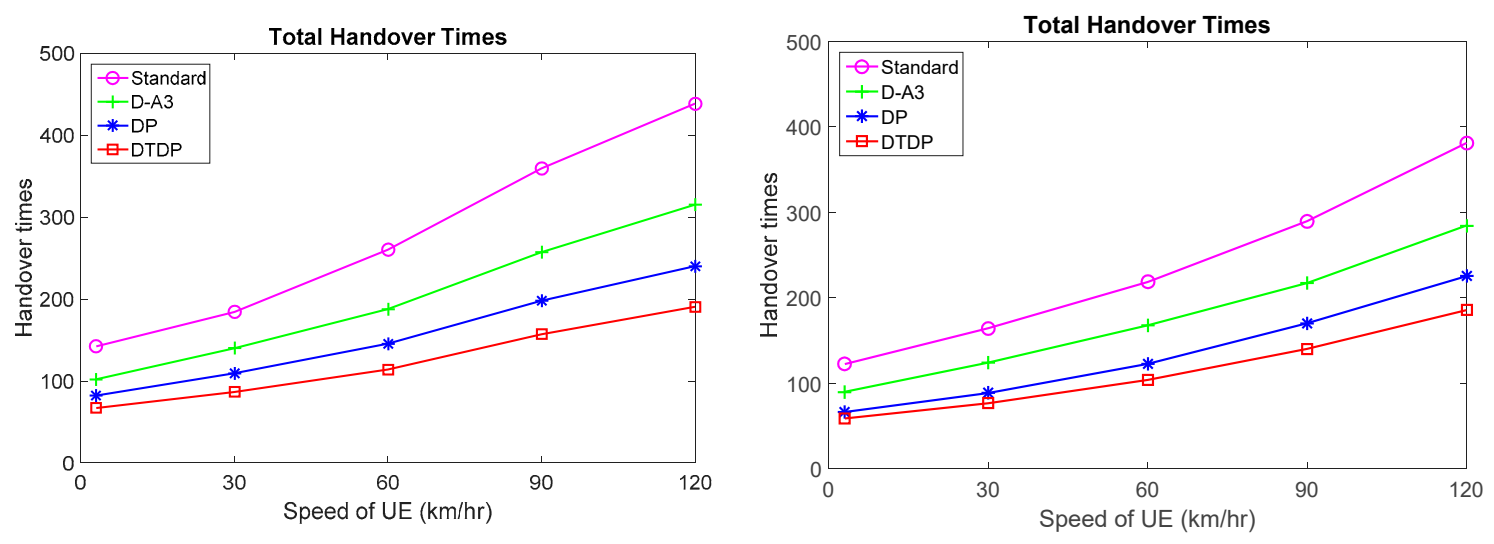

(a)
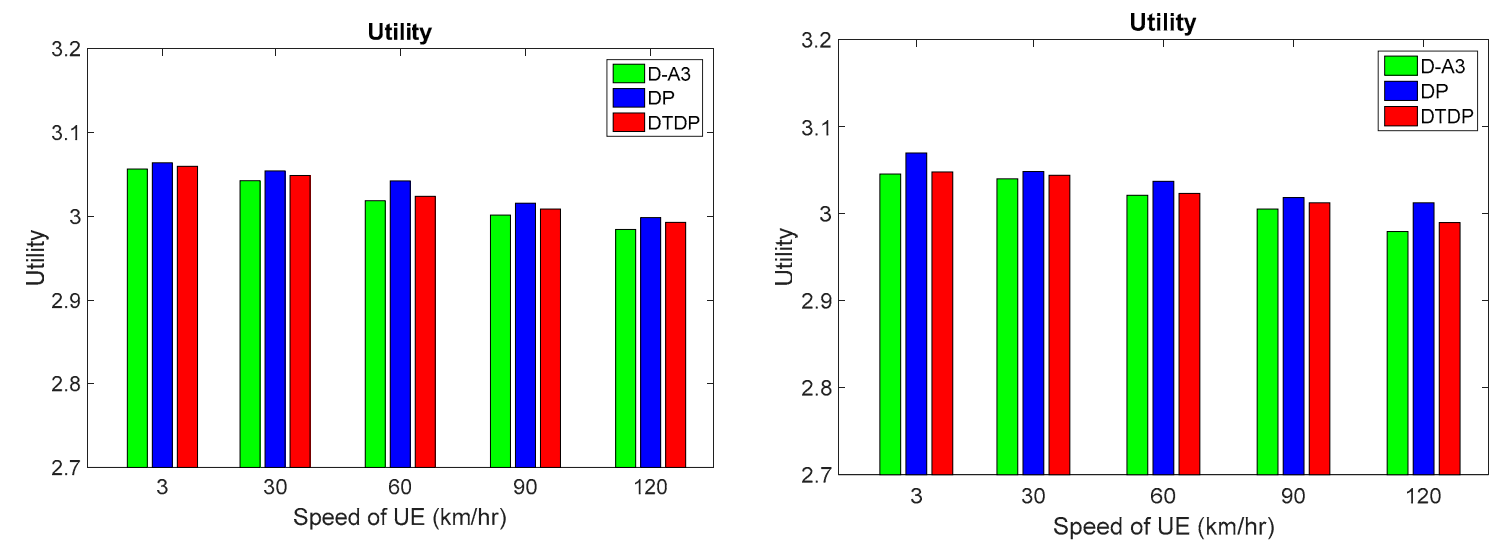

(b)
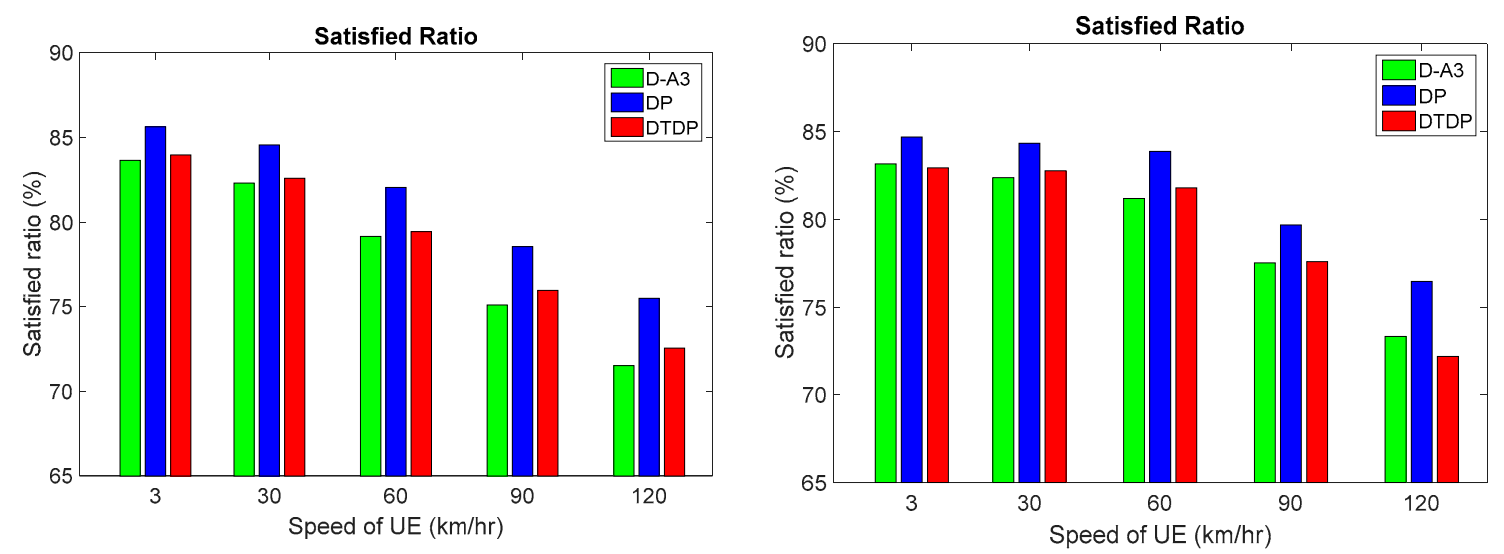

(c)

Figure 5. Total number of handovers, utility, and satisfied ratio versus the moving speed of each D2D user under different mobility models. The charts on the left and the charts on the right correspond to the FD model and RPGM model, respectively. (a) Total number of handovers; (b) utility; (c) satisfied ratio. 
Figure $5 b, c$ shows the average utility value per D2D pair and the satisfied ratio of the system vs. different speeds of users. Here, we can observe that there is a close correlation between the average utility value per D2D pair and the satisfied ratio of the system, as mentioned before. In addition, the average utility value and the satisfied ratio decrease with the increase of the user velocity. This is due to the fact that, as users move rapidly, the frequent handovers caused by unstable RSRPs may happen for each D2D pair, which leads to the lower average utility value. For the comparison of various methods, as mentioned before, since the D-A3 method only chooses the target eNB that has the best RSRP to either one of the DUEs of the same pair. The other DUE may have a worse RSRP value to the target, which results in worse utility than both of the proposed methods (DP and DTDP). DP only considers the utility values for choosing the target eNB and making the handover decision. Consequently, DP incurs additional handovers and generates a longer latency. However, the performance of DP would be better than DTDP in terms of average utility and the satisfied ratio of the system. For the comparison of the two mobility models, the FD model has the larger difference in RSRPs between both DUEs of the same pair, which induces more handovers due to relatively unstable received signal conditions for each D2D pair but it does not really affect the average utility value of the DTDP because, for the utility function, both DUEs' RSRPs are averaged. Similarly, since the satisfied ratio mainly pertains to the average utility, it would not be affected by different mobility models.

Figure 6a illustrates the impacts of the distance between the two DUEs of the same pair on the average handover time and the average throughput. The handover time is calculated as the total time for both DUEs of the same pair to handover to their targets. Note that the time spent for each user to conduct its handover process is set to a fixed value $(40 \mathrm{~ms})$. The delay caused by other procedures include waiting time for a DUE to complete its handover process when the other has done, the transmission time for the original serving eNB to transmit the handover commands to both DUEs, and the transmission time for both DUEs to send the successful-handover messages to the target eNB. Thus, for Figure $6 \mathrm{a}$, as we can see that the average handover time increases with the increase of the distance between the two DUEs of a D2D pair due to the increased waiting time for both DUEs to complete their handover procedures and the increased transmission delay. Also, we can see that the average handover time has a negative effect on the average throughput. This is because, in addition to the decreasing of throughput due to the lower channel gain caused by the increasing distance between two DUEs, the blocking of data transmission between two DUEs during their handover processes would also degrade the throughput. For the comparison of different methods, the conventional standard has the highest probability of letting both DUEs handover to their targets in a non-simultaneous way. Thus, the time consumption in waiting for both DUEs to complete their handover procedures becomes the largest among all methods. As a result, it has the highest average handover time. For D-A3, it is better than the conventional standard in terms of average handover time owing to the choosing of the same target eNB for both DUEs. Since our proposed method chooses the same target for two DUEs and also jointly conducts their handover procedures, DTDP has the lowest average handover time.

Figure $6 \mathrm{~b}$ shows the cumulative distribution function $(\mathrm{CDF})$ of the control signal transmission delay and the average throughput. For the control signal transmission, we consider two different cases. The first one is that both DUEs of the same pair connect to the same eNB for their control signals transmission. The other one is that DUEs connect to different eNBs, which is possible when such a D2D pair is moving around at the cell boundary. As mentioned before, the latter has additional latency due to the information exchanges on the $\mathrm{X} 2$ interface among different eNBs and among eNBs and D2D devices. Therefore, in this aspect, we can observe that there is higher transmission latency for both DUEs of the same pair connected to different eNBs than connected to the same eNB. Furthermore, as we can see from the average throughput when the two DUEs connect to different eNBs, their control channels have higher transmission latency. This also induces lower throughput. 

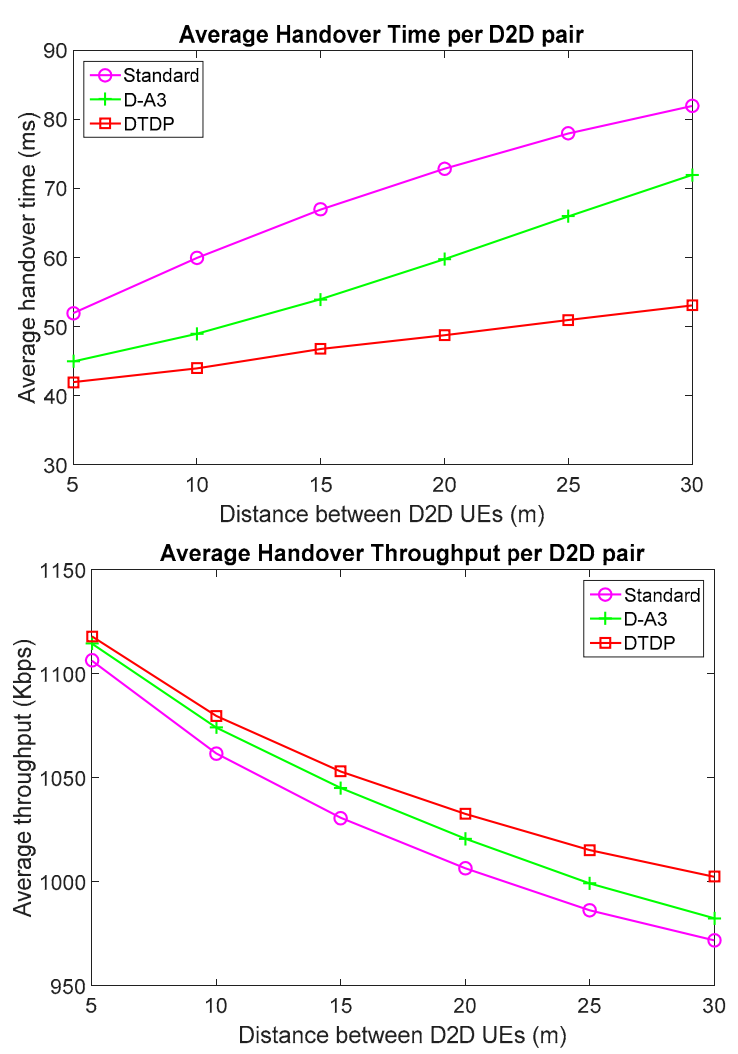

(a)
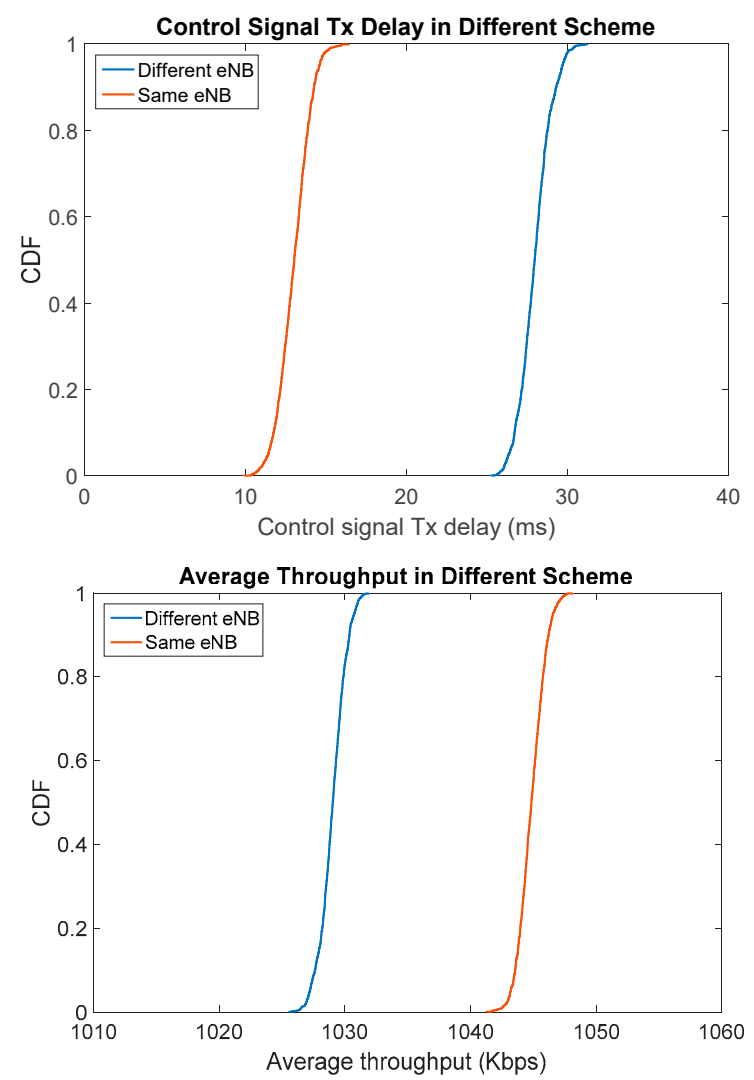

(b)

Figure 6. Average handover time and average throughput versus the distance between two device-to-device user equipment (DUEs) of the same pair, and the cumulative distribution function (CDF) analysis of transmission delay and average throughput of the system. (a) Performance of average handover time and average throughput. (b) CDF of transmission delay and average throughput.

Another issue that deserves further investigation is the number of D2D pairs having both parties connected to the same eNB. To reduce the number of unnecessary handovers, DP and DTDP are designed to let both parties of a D2D pair conduct handover together. As a result, the signaling cost can be minimized. In the next experiment, non-simultaneous handover is allowed for D-A3, denoted by ID-A3. Figure 7 reports the ratio of D2D pairs having both users connected to the same eNB. Both DP and DTDP have a ratio of one. On the other hand, ID-A3 has a ratio of around $70 \%$. It implies that the signaling cost of ID-A3 will be higher than that of DP and DTDP.

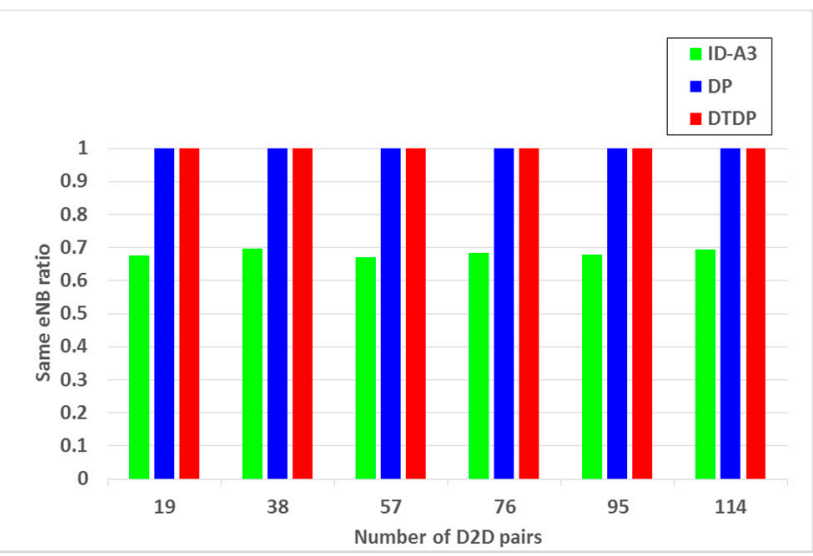

Figure 7. Ratio of D2D pairs having parties connected to the same eNB. 
Previous simulations present the system dynamics in the control plane, so now we turn our attention to the data plane. For 76 D2D pairs moving at a speed of $3 \mathrm{~km} / \mathrm{h}$, we vary the transmission power of UEs and observe the change in system throughput. Figure 8 shows that the system throughput is correlated to the UE transmission power. For the same distance between two UEs, the higher transmission power at the sender implies the higher received signal strength at the receiver. As indicated in Equations (3) and (4), the higher received signal strength will result in the higher system capacity and, therefore, the higher system throughput. It is worth noting that the change in UE transmission power will not affect the number of handovers, utility function, and satisfaction ratio. That is because, for those handover management schemes that we considered, the selection of handover target and the timing of handover rely on the RSRP in the control plane rather than the UE transmission power in the data-plane.

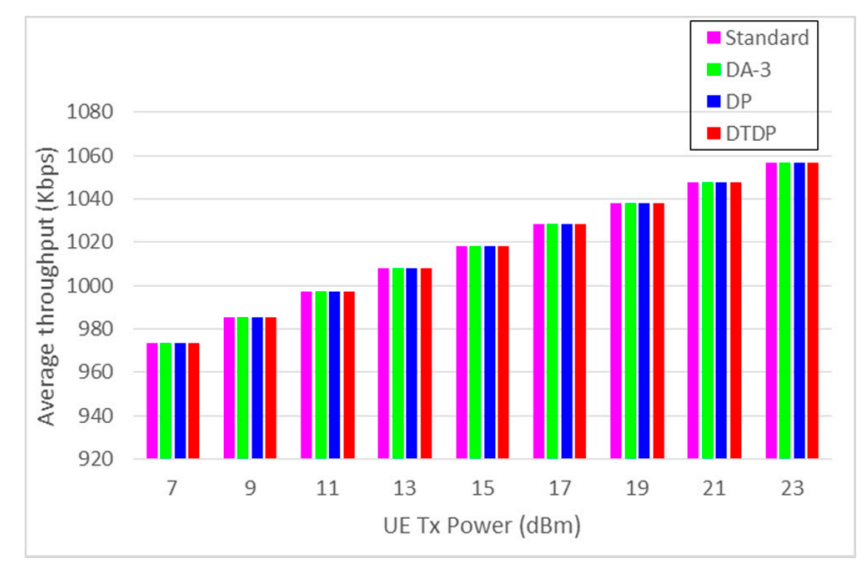

Figure 8. System throughput versus user equipment (UE) transmission power.

\subsection{Micro View of Numerical Results}

The numerical results described above are evaluated from a macro view of our simulations. In this section, we will focus on the micro view of our system. In other words, we will look into the detailed behavior of a single instance of the D2D pair. The first pair of D2D users in the system is chosen as the object of observation, and the comparisons between various methods are made based on the performance of this particular D2D pair.

Figure 9a shows the number of handovers for the first pair of D2D users versus their moving speed. As mentioned in the previous section, when the moving speeds of both users increase, there is a higher probability for the D2D pair to pass the overlapped areas of multiple eNBs. This results in more number of handovers. Additionally, the results of comparison among different methods are the same as before. DTDP chooses a single eNB as the handover target of the D2D pair and makes the handover decision weighing both DUEs' movements and their received signal conditions. Doing so can greatly reduce unnecessary handovers.

In Figure 9b,c, the utility value and the average satisfied ratio for the first D2D pair in the system versus its moving speed are given. As discussed in the previous section, with the increasing of the speeds of both DUEs, the received signal conditions of both users become more unstable. Thus, it may be possible for a rapid-moving pair of users to start their handover process before they have left the original serving eNB's coverage. This will give a lower utility value. The utility value is correlated to the satisfied ratio. With the increasing speeds of both DUEs, the satisfied ratio will decrease accordingly. For the comparison between various methods, DP only depends on received signal conditions so as to have the highest utility value and satisfied ratio among all the methods. However, extra handovers may also degrade the performance. For D-A3, since it only chooses the target eNB that has the best RSRP to either one of the DUEs, the other DUE may have inferior RSRP to the target. This behavior results in the worst utility and satisfied ratio compared with other methods. As for DTDP, with the 
considerations of received signal conditions for both DUEs and their movements, its utility value and satisfied ratio are a little bit worse than DP. However, it would introduce fewer handovers and achieve the best performance in terms of transmission latency and average throughput.

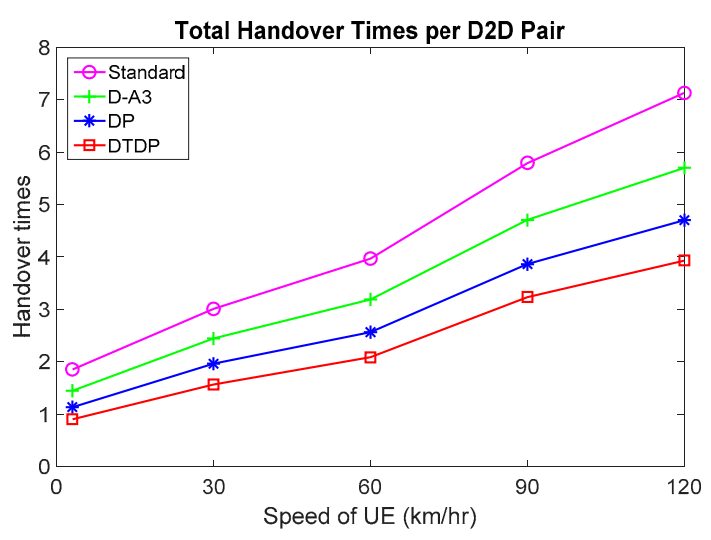

(a)

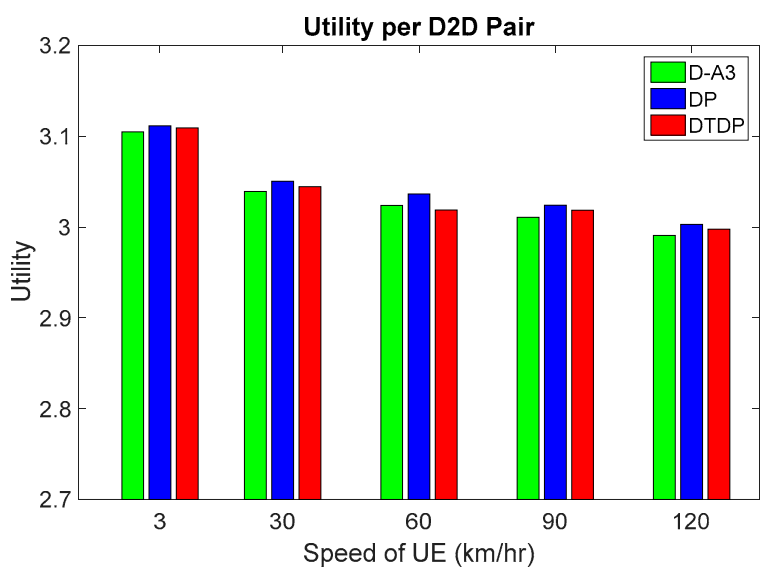

(b)

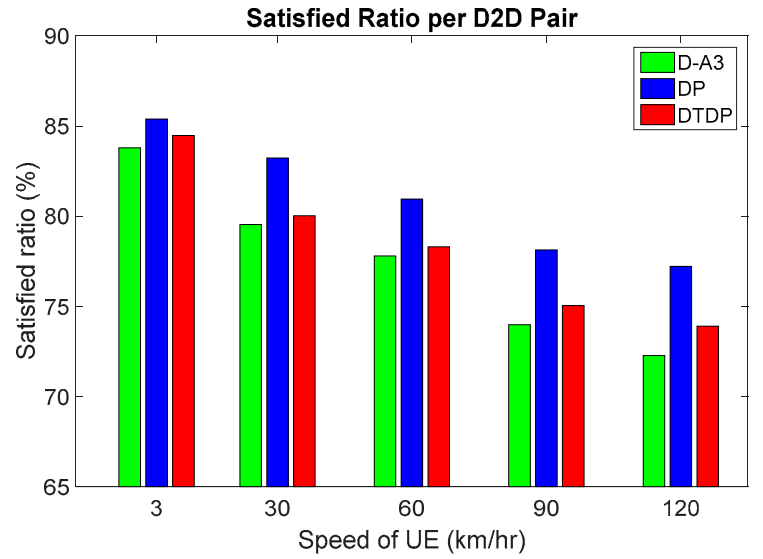

(c)

Figure 9. Total number of handovers, utility, and average satisfied ratio for the first D2D pair in the system versus its moving speed. (a) Total number of handovers for the first D2D pair. (b) Utility for the first D2D pair. (c) Average satisfied ratio for the first D2D pair.

\section{Conclusions}

In this paper, we have proposed a novel handover mechanism for D2D communications. First, a list of handover candidate eNBs is generated for each D2D pair based on both DUEs' movements and received signal conditions. With the introduction of $D_{\text {counter }}$ and the utility function, we can then determine the best handover target for this D2D pair. The decision jointly considers their historical movements and the received signal conditions. Second, we take into account both DUEs' satisfaction in terms of their received signal conditions with respect to their original and target serving eNBs to decide whether or not to hand over them to the target eNB simultaneously. As in the result, in addition to reducing handovers and decreasing transmission latency, the better performance in terms of received signal quality for both DUEs is also achieved.

According to the simulation results, the proposed DTDP method shows better performance than the conventional standard and D-A3 method in terms of the total number of handovers, average utility value, and satisfied ratio, both at the system level and user level. Although the DP method, which does not account for $D_{\text {counter }}$, shows better performance in the average utility value and satisfied ratio, it incurs more handovers and lengthens transmission latency, which leads to lower throughput. 
Author Contributions: Conceptualization, W.K.L. and F.-S.C.; software, F.-S.C. and M.-H.S.; validation, C.-S.S.; writing-original draft preparation, C.-Y.H. and C.-S.S.; writing-review and editing, W.K.L. and C.-S.S.; supervision, W.K.L. All authors have read and agreed to the published version of the manuscript.

Funding: This research was funded by MOST of Taiwan, under the grant number 108-2221-E-110-017-.

Conflicts of Interest: The authors declare no conflict of interest.

\section{References}

1. 3GPP. LTE Device to Device (D2D) Proximity Services (ProSe); UE Radio Transmission and Reception. 3rd Generation Partnership Project (3GPP), TR 36.877. March 2015. Available online: http://www.3gpp.org/ftp/ Specs/html-info/36877.htm (accessed on 25 June 2020).

2. Chen, X.; Kim, M.J.; Yoo, S.H.; Park, N.Y.; Youn, H.Y. Efficient and prompt handover in LTE-based systems by predicting the target eNodeBs. In Proceedings of the 2014 International Conference on Cyber-Enabled Distributed Computing and Knowledge Discovery, Shanghai, China, 13-15 October 2014; pp. 406-413.

3. Lee, C.; Shin, S.; Chung, J.M. Enhanced LTE handover scheme using NFV for LTE handover delay reduction. In Proceedings of the 2016 IEEE International Conference on Consumer Electronics-Asia (ICCE-Asia), Seoul, Korea, 26-28 October 2016; pp. 1-2.

4. Agrawal, J.; Mor, P.; Keller, J.M.; Patel, R.; Dubey, P. Introduction to the basic LTE handover procedures. In Proceedings of the 2015 International Conference on Communication Networks (ICCN), Gwalior, India, 19-21 November 2015; pp. 197-201.

5. Tomasov, G.; Wu, M.; Wen, J.; Liu, H. LTE fixed-point handover algorithm for high-speed railway scenario. In Proceedings of the 2013 3rd International Conference on Computer Science and Network Technology, Dalian, China, 12-13 October 2013; pp. 919-923.

6. 3GPP. Evolved Universal Terrestrial Radio Access (E-UTRA); Radio Resource Control (RRC). 3rd Generation Partnership Project (3GPP), TS 36.331. December 2015. Available online: http://www.3gpp.org/ftp/Specs/ html-info/36331.htm (accessed on 25 June 2020).

7. Dimou, K.; Wang, M.; Yang, Y.; Kazmi, M.; Larmo, A.; Pettersson, J.; Muller, W.; Timner, Y. Handover within 3GPP LTE: Design principles and performance. In Proceedings of the 2009 IEEE 70th Vehicular Technology Conference Fall, Anchorage, AK, USA, 20-23 September 2009; pp. 1-5.

8. 3GPP. Evolved Universal Terrestrial Radio Access Network (E-UTRAN); X2 Application Protocol (X2AP). 3rd Generation Partnership Project (3GPP), TR 36.423. March 2015. Available online: http://www.3gpp.org/ $\mathrm{ftp} /$ Specs/html-info/36423.htm (accessed on 25 June 2020).

9. Priyadharshini, A.S.; Bhuvaneswari, P.T.V. A study on handover parameter optimization in lte-a networks. In Proceedings of the 2016 International Conference on Microelectronics, Computing and Communications (MicroCom), Durgapur, India, 23-25 January 2016; pp. 1-5.

10. Kollias, G.; Adelantado, F.; Verikoukis, C. The impact of inter-site distance and time-to-trigger on handover performance in LTE-A HetNets. In Proceedings of the 2015 IEEE International Conference on Communications (ICC), London, UK, 8-12 June 2015; pp. 3969-3974.

11. Wang, Y.H.; Chang, J.L.; Huang, G.R. A handover prediction mechanism based on LTE-A UE history information. In Proceedings of the 2015 18th International Conference on Network-Based Information Systems, Taipei, Taiwan, 2-4 September 2015; pp. 167-172.

12. Chang, F.M.; Wan, H.I.; Hu, S.Y.; Kao, S.J. An efficient handover mechanism by adopting direction prediction and adaptive time-to-trigger in LTE networks. In Computational Science and Its Applications (ICCSA); Springer: Berlin/Heidelberg, Germany, 2013; pp. 270-280.

13. Cheikh, A.B.; Ayari, M.; Langar, R.; Pujolle, G.; Saidane, L.A. Optimized handoff with mobility prediction scheme using hmm for femtocell networks. In Proceedings of the 2015 IEEE International Conference on Communications (ICC), London, UK, 8-12 June 2015; pp. 3448-3453.

14. Sathya, V.; Ramamurthy, A.; Kumar, S.S.; Tamma, B.R. On improving SINR in LTE HetNets with D2D relays. Comput. Commun. 2016, 83, 27-44. [CrossRef]

15. Ouali, K.; Kassar, M.; Nguyen, T.M.T.; Sethom, K.; Kervella, B. Modeling D2D handover management in $5 \mathrm{~g}$ cellular networks. In Proceedings of the 2017 13th International Wireless Communications and Mobile Computing Conference (IWCMC), Valencia, Spain, 26-30 June 2017; pp. 196-201. 
16. Ouali, K.; Kassar, M.; Sethom, K. Handover performance analysis for managing D2D mobility in 5G cellular network. IET Commun. 2018, 12, 1925-1936. [CrossRef]

17. Yilmaz, O.N.C.; Li, Z.; Valkealahti, K.; Uusitalo, M.A.; Moisio, M.; Lunden, P.; Wijting, C. Smart mobility management for D2D communications in $5 \mathrm{~g}$ networks. In Proceedings of the 2014 IEEE Wireless Communications and Networking Conference Workshops (WCNCW), Istanbul, Turkey, 6-9 April 2014; pp. 219-223.

18. Li, Y.; Su, Z.; Huang, L.; Song, W. A speed-aware joint handover approach for clusters of D2D devices. In Proceedings of the 2018 IEEE 88th Vehicular Technology Conference (VTC-Fall), Chicago, IL, USA, 27-30 August 2018; pp. 1-5.

19. Chen, H.Y.; Shih, M.J.; Wei, H.Y. Handover mechanism for device-to-device communication. In Proceedings of the 2015 IEEE Conference on Standards for Communications and Networking (CSCN), Tokyo, Japan, 28-30 October 2015; pp. 72-77.

20. Ouali, K.; Kassar, M.; Nguyen, T.M.T.; Sethom, K.; Kervella, B. An efficient D2D handover management scheme for SDN-based 5G networks. In Proceedings of the 2020 IEEE 17th Annual Consumer Communications \& Networking Conference (CCNC), Las Vegas, NV, USA, 10-13 January 2020; pp. 1-6.

21. Shannon, C.E. A mathematical theory of communication. Bell Syst. Tech. J. 1948, 27, 379-423. [CrossRef]

22. 3GPP. Evolved Universal Terrestrial Radio Access (E-UTRA); Further Advancements for E-UTRA Physical Layer Aspects. 3rd Generation Partnership Project (3GPP), TR 36.814. March 2010. Available online: http://www.3gpp.org/ftp/Specs/html-info/36814.htm (accessed on 25 June 2020).

23. Hong, X.; Gerla, M.; Pei, G.; Chiang, C.C. A group mobility model for ad hoc wireless networks. In Proceedings of the 2nd ACM International Workshop on Modeling, Analysis and Simulation of Wireless and Mobile Systems (MSWiM), Seattle, WA, USA, 15-19 August 1999; pp. 53-60.

(C) 2020 by the authors. Licensee MDPI, Basel, Switzerland. This article is an open access article distributed under the terms and conditions of the Creative Commons Attribution (CC BY) license (http://creativecommons.org/licenses/by/4.0/). 